\title{
Unexpected one-pot synthesis of 2,4-dialkylthiopentanedials by the reaction of alkylthioacetic aldehydes with dihalomethane and diethylamine
}

\author{
Natalia A. Keiko,* Ludmila G. Stepanova, Ekaterina A. Verochkina, \\ Ludmila I. Larina, and Yurii A. Chuvashev
}

\begin{abstract}
A.E. Favorsky Irkutsk Institute of Chemistry, Siberian Branch of the Russian Academy of Sciences, 664033 Irkutsk, Russian Federation

E-mail: keiko@irioch.irk.ru
\end{abstract}

\begin{abstract}
The reaction of alkylthioacetic aldehydes with dihalomethane and diethylamine has allowed the unexpected domino sequence of three reactions (Mannich - dehydroamination - Michael) to be revealed. A facile one-pot three-step protocol for the synthesis of 2,4-dialkylthio pentanedials in up to $65 \%$ yield has been developed on the basis of this reaction. A biological activity of one pentadial has been determined for the first time. The activity of alkylthioacetic aldehydes as a $\mathrm{CH}$-acids is shown in two anion reactions.
\end{abstract}

Keywords: Aldehydes, carbanions, domino reaction, Mannich and Michael additions

\section{Introduction}

It has been reported a variant of the aminomethylation reaction (Mannich reaction), where the interaction of $\alpha-\mathrm{ArCH}_{2} \mathrm{O}$ - and $\alpha-\mathrm{R}_{2} \mathrm{~N}$-substituted aldehydes with dihalomethane and diethylamine leads to the corresponding 2-functional substituted propenals. ${ }^{1}$ However, $\alpha$ methylenation reactions of $\alpha$-alkylthio substituted aldehydes have not yet been performed. Meanwhile, the introduction of a sulfur-containing substituent in the $\alpha$-position to a carbonyl group is assumed to enhance the thermodynamic acidity of an adjacent proton by factor of $10^{3}$ as compared with a simple ketone as well as to stabilize an anion at the carbon neighboring sulfur. ${ }^{2}$

The study was initially aimed at application of the mentioned method to synthesize 2-RSpropenals from alkylthioacetic aldehydes as $\mathrm{CH}$-acids. A while ago, we have obtained 2buthylthiopropenal by other protocol starting from formaldehyde. ${ }^{3}$ The use of iminium salts is supposed to substantially raise the yields of aminomethylation products compared to conventional Mannich reaction in formaldehyde and dialkylamine aqueous solution. Besides, this facilitates the procedure. ${ }^{4}$ It is pertinent to note that 2-alkylthiosubstituted propenals are strongly 
prone to very rapid cyclodimerization through Diels-Alder reaction. ${ }^{5,6}$ The rate of the reaction of 2-butylthiopropenal is $10^{4}$ times higher than that of 2-ethoxypropenal. ${ }^{7}$ The other routes to 2alkylthiopropenals result also in the formation of 2,5-dialkylthio-2,3-dihydro-2-formyl-4Hpyrans exclusively. ${ }^{8}$ For this reason, the latter should be the only products of Mannich reaction performed by the experimental procedure ${ }^{1}$ (Scheme 1).

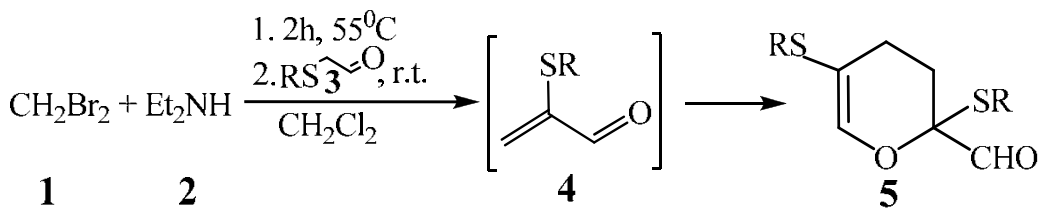

\section{Scheme 1}

\section{Results and Discussion}

Contrary to expectations, the interaction of 2-butylthioacetic aldehyde with dibromomethane and diethylamine at thorough implementation of the protocol proposed for 2-phenyl methoxypropenal ${ }^{1}$ has not resulted in 2-butylthiopropenal 4a (Scheme 2).

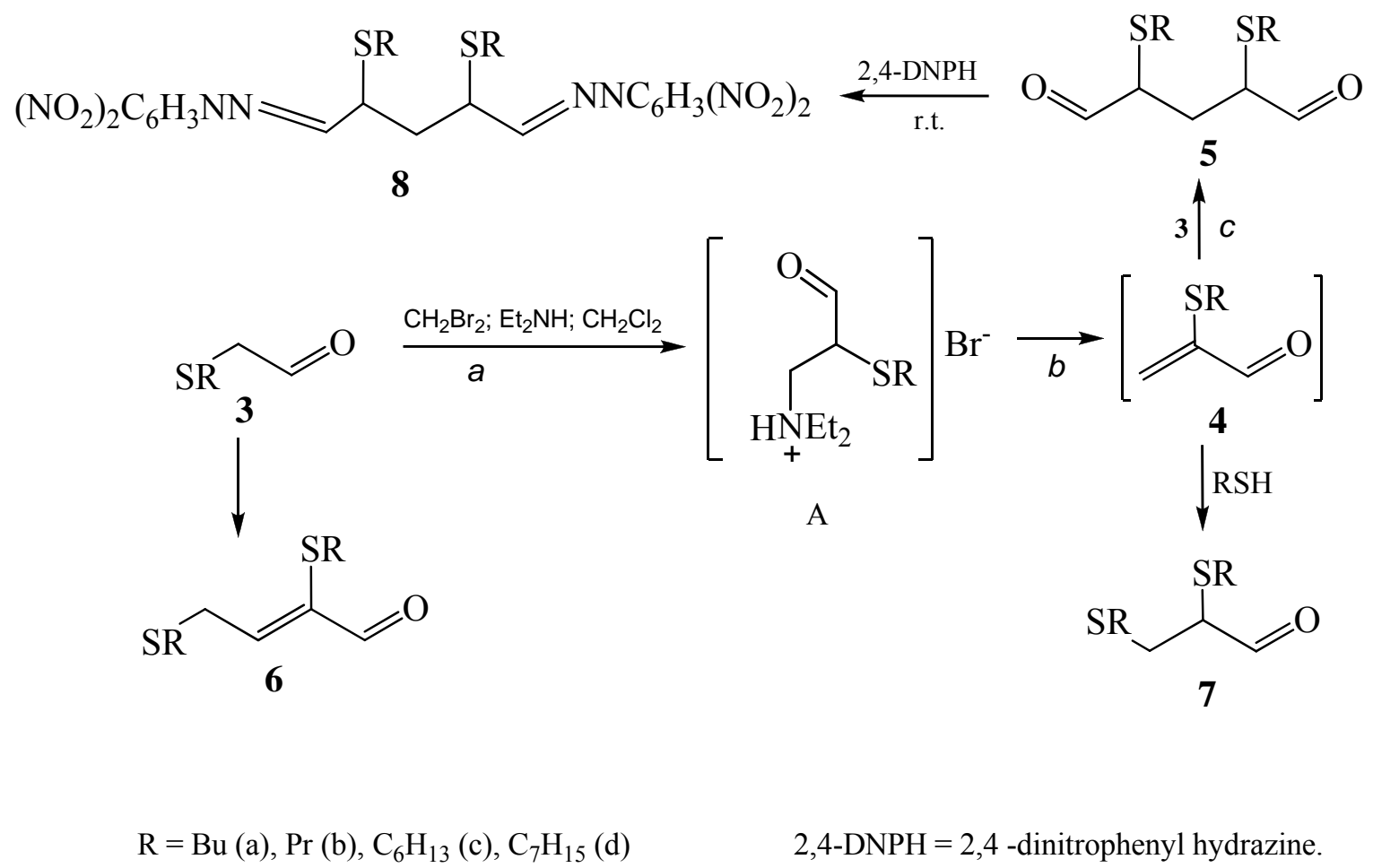

Scheme 2. Postulated mechanisms of novel sequence of domino reactions $(\boldsymbol{a}, \boldsymbol{b}, \boldsymbol{c})$ for synthesis of the title compounds $\mathbf{5}$ and generation of by-products. 
Instead, 2,4-dibutylthio pentanedial 5a has been obtained in the yield $40 \%$ (Table 1, entry 1). The attempts to adapt the method in order to obtain $\alpha$-butylthiopropenal 4 a have not made the aim closer but produced much of various by-products by competitive reactions.

Table. Comparative yields and reaction conditions used for synthesis of 5a

\begin{tabular}{|c|c|c|c|c|c|c|c|}
\hline Entry & $\begin{array}{c}2 \\
\text { mmol } \\
\end{array}$ & $\begin{array}{c}1 \\
\mathrm{mmol} \\
\end{array}$ & $\begin{array}{c}\mathrm{BuSCH}_{2} \mathrm{CHO} \\
\mathrm{mmol}\end{array}$ & $\begin{array}{c}\mathrm{CH}_{2} \mathrm{Cl}_{2} \\
\mathrm{~mL}\end{array}$ & $\begin{array}{c}\text { Molar ratio } \\
\mathbf{1}: \mathbf{2}: \mathbf{3 a} \\
\end{array}$ & $\begin{array}{l}\text { Time }^{\mathrm{a}} \\
(\mathrm{h})\end{array}$ & $\begin{array}{l}\text { Yield of } \\
\mathbf{5 a}(\%)^{\mathrm{b}}\end{array}$ \\
\hline 1 & 45 & 9 & 3 & $\ldots$ & $15: 3: 1$ & 2 & 40 \\
\hline 2 & 22.5 & 4.5 & 7 & 6 & $3.2: 1: 1.6$ & 3 & 40 \\
\hline 3 & 22.5 & 4.5 & 5 & 6 & $4.5: 1: 1.2$ & 3,5 & 45 \\
\hline 4 & 110 & 22 & 11 & 22 & $10: 2: 1$ & $\begin{array}{c}2.520{ }^{\circ} \mathrm{C} \\
\text { 5days } 8{ }^{\circ} \mathrm{C}\end{array}$ & 88 \\
\hline 5 & 74 & 14.8 & 7.4 & 6 & $10: 2: 1$ & 12 & 87 \\
\hline
\end{tabular}

${ }^{\text {a. }}$ On r.t.

${ }^{\mathrm{b}}$ Yield was determined by ${ }^{1} \mathrm{H}$ NMR.

A change in initial reagents ratio results in a decrease in the reaction selectivity. Thus, the reduction of diethylamine : aldehyde 3 initial ration to 1,2-1,6:1 (entries 2-3) causes the formation of many impurities (GC - MS). Among them are detected the product of crotonic auto condensation of butylthioacetic aldehyde 6 (up to $9 \%$ ), adduct of hydrosulfanylation of the intermediate enal 4a - 2,3-dibutylthiopropanal 7 (up to $5 \%$ ) and radical splitting products of compounds 4a - 6. The latter include butanethiol along with the corresponding disulfide and some others.

Varying the reaction conditions (see Table) allows the limitations of the process implementation to be determined. The yield of product 5a has reached $87 \%\left({ }^{1} \mathrm{H}\right.$ NMR), while after column chromatography isolation it drops to $65 \%$. The reactions of different alkylthioacetic aldehydes $\mathbf{3}$ b-d under optimized conditions leading to $\mathbf{5} \mathbf{b}-\mathbf{d}$ in excellent yield $\left(87 \%,{ }^{1} \mathrm{H}\right.$ NMR) confirm these results. The formation of dials $\mathbf{5}$ is shown in Scheme 2.

Apparently, the interaction of diethylamine with 1,1-dibromomethane leads to in situ formation of an active complex similar to $\left[\mathrm{Et}_{2} \mathrm{~N}^{+}=\mathrm{CH}_{2}\right] \mathrm{Br}^{-9}$ or $\left[\mathrm{Et}_{2} \mathrm{~N}_{-} \mathrm{CH}_{2} \mathrm{NEt}_{2}\right] \cdot 2 \mathrm{HBr}{ }^{1}$ which further delivers the Mannich base $\mathbf{A}$ in the reaction with activated molecule of alkylthioacetic aldehyde 3. Eliminating diethylamine, the Mannich base is transformed into enal 4. The unreacted initial aldehyde 3 adds to enal $\mathbf{4}$ by Michael reaction (c). Interestingly, the reaction $\boldsymbol{c}$ is so fast that the intermediate 4 turns to be hardly detectable by ${ }^{1} \mathrm{H}$ NMR. In the sequence of the three reactions $(a, b, c)$, the Michael reaction efficiency is seemingly due to the participation of 
active ammonium complex prepared for the Mannich reaction. Thus, the studied domino reaction presents a new example of competitive tandem catalysis. ${ }^{10}$ In this study, it has been shown for the first time that alkylthioacetic aldehyde can be involved in two successive stages and appears to be active $\mathrm{CH}$-acid. The Mannich - aza-Michael reactions sequence was only described before. $^{11}$

For the selective synthesis of dials 5 one needs to perform the reaction at the initial ratio of $\mathrm{CH}_{2} \mathrm{Br}_{2}: \mathrm{Et}_{2} \mathrm{NH}: 3$ equal to $10: 2: 1$, room temperature and for $12 \mathrm{~h}$. Under such conditions, the conversion of 3 reaches $100 \%$ (according to ${ }^{1} \mathrm{H}$ NMR) and the content of by-products is the lowest. ${ }^{1} \mathrm{H}$ NMR spectra of dials $\mathbf{5}$ allows them to be identified as a mixture of diastereoisomers in the ratio 1:1-2.

The structure of dials $\mathbf{5 a , b}$ is evidenced from the formation of bis-2,4-dinitrophenyl hydrazones 8a,b (Scheme 2) synthesized by the reaction of 5a,b with 2,4-dinitrophenyl hydrazine (2,4-DNPH) using the method for a dicarbonyl substances. ${ }^{12}$ The ratio of diasteriomeres $\mathbf{8 a}, \mathbf{b}$ is $1: 1,5$.

The obtained dialkylthiosubstituted dials $\mathbf{5}$ might find an application as very efficient antimicrobial prodrugs, since their unsubstituted analogue, glutaric dialdehyde, is widely known in medicine as a microbicide ${ }^{13}$ and disinfectant,${ }^{14}$ it is also a high-effective crosslinking agents, ${ }^{15}$ and co-monomer. ${ }^{16}$ We have shown that dial 5a exhibits the pronounced antimicrobial activity (for Staphilococcus Aureus minimal inhibition concentration (MIC) $-15 \mu \mathrm{g} / \mathrm{mL}$, minimal bactericide concentration $31 \mu \mathrm{g} / \mathrm{mL}$; for E. Coli - MIC $31 \mu \mathrm{g} / \mathrm{mL}$, minimal bactericide concentration $-62 \mu \mathrm{g} / \mathrm{mL}$ ).

\section{Conclusions}

In summary, the Mannich and Michael reactions are known to be the most versatile and applicable reactions to furnish selectively the carbon-carbon bonds. ${ }^{11,}{ }^{17}$ In this paper, we have shown that the unexpected domino sequence of these reactions starting from alkylthioacetic aldehydes as $\mathrm{CH}$-acids, diethylamine and dihalomethane leads to 2,4-dialkylthio pentanedials. The dehydroamination of the intermediate $\beta$-diethylamine aldehyde is a middle stage of this process. The catalytic complex prepared for the Mannich reaction according to the method ${ }^{1}$ appears to be very active also for the successive Michael reaction. This activity causes the higher rate of the Michael reaction as compared to the Mannich reaction. As a result, 2-alkylthio propenal generated in situ is not accumulated the in reaction medium, but enter rapidly into the Michael reaction. Hence the studied reaction sequence is a new example of competitive tandem catalysis.

\section{Experimental Section}

General Procedure. ${ }^{1} \mathrm{H}$ and ${ }^{13} \mathrm{C}$ NMR spectra recorded on a Bruker DPX 400 spectrometer (400.13 and $100.61 \mathrm{MHz}$ accordingly) using $\mathrm{CDCl}_{3}$ as a solvent, and HMDS as an internal standard. GC - MS analysis was performed using Hewlett-Packard 5971A mass-selective 
detector (electron impact, $70 \mathrm{eV}$ ) coupled with an HP 5890 gas chromatograph (Ultra-2 column, $5 \%$ of phenylmethylsilicone; injector temperature $250{ }^{\circ} \mathrm{C}$; oven temperature 70 to $280{ }^{\circ} \mathrm{C}$; at rate of $20{ }^{\circ} \mathrm{C} \mathrm{min}^{-1}$ ). IR spectra were recorded in $\mathrm{KBr}$ on a Specord 75 IR spectrophotometer. Elemental analysis were carried out in a Thermo Finnigan automatic analyzer 1112 ser. Column chromatographic separations were done on silica gel 60 (70-200 mesh). Melting points were obtained on a Micro-Hot-Stage Poly Therm A instrument (Wagner and Muzn) and are uncorrected.

\section{General procedure for the synthesis of 2,4-dialkylthio pentanedials 5a-d}

A solution of diethylamine $(14.8 \mathrm{mmol})$ in dibromomethane $(74 \mathrm{mmol})$ was heated at $55{ }^{\circ} \mathrm{C} 1.5$ $\mathrm{h}$. Then the reaction mixture was cooled to room temperature and added to the solution of alkylthioacetic aldehyde $3(7.4 \mathrm{mmol})$ in dichloromethane $(14.6 \mathrm{~mL})$. The reaction was monitored by TLC on silica gel plates $\left(\right.$ Silufol ${ }^{R}$ ) (eluent: acetonitrile - hexane - diethyl ether 1:4:1), NMR spectroscopy and/or gas chromatography. On next day, when the starting material 3 was totally consumed, the reaction mixture was washed with acid water, dried $\left(\mathrm{MgSO}_{4}\right)$ and the solvent was evaporated under reduced pressure. The residue was distillated in high vacuum. On isolating by vacuum distillation the dials $\mathbf{5} \mathbf{c}, \mathbf{d}$ were tarified. However, they could be isolated by column chromatography on silica gel (70-200 mesh, eluent - acetonitrile or hexane:diethyl ether 2:1).

2,4-Dibutylthio pentanedial (5a). Yield is up to $65 \%$ after silica gel chromatography. Yellowish oil that boils undecomposed during molecular and even vacuum distillation, bp 180 ${ }^{\circ} \mathrm{C} / 1 \mathrm{~mm} \mathrm{Hg} .{ }^{1} \mathrm{H}$ NMR $\left(\mathrm{CDCl}_{3}\right): \delta 0.91\left(\mathrm{t}, J=7.3 \mathrm{~Hz}, 6 \mathrm{H}, \mathrm{CH}_{3}\right.$ in $\left.\mathrm{Bu}\right), 1.38\left(\mathrm{~m}, 4 \mathrm{H}, \mathrm{C}_{2} \mathrm{CH}_{3}\right)$, $1.52\left(\mathrm{~m}, 4 \mathrm{H}, \mathrm{C}_{2} \mathrm{CH}_{2} \mathrm{CH}_{3}\right), 2.08\left(\mathrm{t},{ }^{3} \mathrm{~J}=7.5 \mathrm{~Hz}, 1 \mathrm{H}, \mathrm{H}_{2} \mathrm{C}-3\right), 2.39\left(\mathrm{~m}, 4 \mathrm{H}, \mathrm{SCH}_{2}\right.$ and $1 \mathrm{H}$, $\left.\mathrm{H}_{2} \mathrm{C}-3\right), 3.42$ and $3.45\left(\mathrm{td},{ }^{3} J=7.5 \mathrm{~Hz},{ }^{3} J=2.5 \mathrm{~Hz}, 2 \mathrm{H}, 2 \mathrm{SC} * \mathrm{H}\right), 9.35\left(\mathrm{~d},{ }^{3} J=2.5 \mathrm{~Hz}, 1 \mathrm{H}\right.$, $\mathrm{CHO}), 9.38\left(\mathrm{~d},{ }^{3} \mathrm{~J}=2.7 \mathrm{~Hz}, 1 \mathrm{H}, \mathrm{CHO}\right) . \mathrm{GC}-\mathrm{MS}: \mathrm{m} / \mathrm{z}(\%)=276(35)[\mathrm{M}]^{+}, 258$ (2), 219 (9) $[\mathrm{M}-\mathrm{Bu}]^{+}, 201$ (4), 191 (9), 173 (2) , 157 (38), 145 (5), 132 (100) [BuSCH$\left.{ }_{2} \mathrm{CHO}\right]^{+}, 117$ (6), 101 (23) $[\mathrm{C} \equiv \mathrm{S}-\mathrm{Bu}]^{+}, 89(14)[\mathrm{BuS}]^{+}, 76(13), 57(28)[\mathrm{Bu}]^{+}, 41(35), 29(23)[\mathrm{CHO}]^{+}$. Anal. Calcd for $\mathrm{C}_{13} \mathrm{H}_{24} \mathrm{~S}_{2} \mathrm{O}_{2}$ (\%): C, 56.51; H, 8.69; S, 23.21. Found: C, 56.56; H, 8.68; S, 22.87. In some experiments 2,3-dibutylthiopropenal 7 described before ${ }^{3}$ and 2,4-dibutylthio-2-butenal 6 were found by GC - MS among the reaction by-products. For the last-named $6 \mathrm{bp} 162{ }^{\circ} \mathrm{C} / 2 \mathrm{~mm} \mathrm{Hg}$. ${ }^{1} \mathrm{H} \mathrm{NMR}\left(\mathrm{CDCl}_{3}\right): \delta 0.90$ and 0.92 (two t, ${ }^{3} \mathrm{~J}=7.3 \mathrm{~Hz}, 6 \mathrm{H}, \mathrm{CH}_{3}$ in $\mathrm{Bu}$ ), $1.42\left(\mathrm{~m}, 4 \mathrm{H}, \mathrm{CH}_{2} \mathrm{CH}_{3}\right.$ ), $1.58\left(\mathrm{~m}, 4 \mathrm{H}, \mathrm{CH}_{2} \mathrm{CH}_{2} \mathrm{CH}_{3}\right), 2.45\left(\mathrm{t},{ }^{3} \mathrm{~J}=7.4 \mathrm{~Hz}, 2 \mathrm{H}, \mathrm{SCH}_{2}\right), 2.83\left(\mathrm{t},{ }^{3} \mathrm{~J}=7.3 \mathrm{~Hz}, 2 \mathrm{H}, \mathrm{SCH}_{2}\right)$, $3.58\left(\mathrm{~d}, J=7.7 \mathrm{~Hz}, 2 \mathrm{H}, \underline{\mathrm{C}}_{2}\right), 6.87$ (t, $\left.J=7.7 \mathrm{~Hz}, 1 \mathrm{H}, \mathrm{HC}=\right), 9.46(\mathrm{c}, 1 \mathrm{H}, \mathrm{CHO})$. MS (70 eV): $\mathrm{m} / \mathrm{z}(\%)=246(42)[\mathrm{M}]^{+}, 217(18)[\mathrm{M}-\mathrm{Et}]^{+}, 189(22)[\mathrm{M}-\mathrm{Bu}]^{+}, 156(33)[\mathrm{M}-\mathrm{BuSH}]^{+}, 133(32)$ $\left[\mathrm{M}-\left(\mathrm{CH}_{2}\right)_{4}\right]^{+}, 113$ (17), 100 (86) [M-BuSH- $\left.\left(\mathrm{CH}_{2}\right)_{4}\right]^{+}, 99$ (100) [M-BuSH-Bu] $]^{+}, 87$ (14), 73 (20) $[\mathrm{S}=\mathrm{C}-\mathrm{CHO}]^{+}, 71(58), 57(26)[\mathrm{Bu}]^{+}$, 41(43). Anal. Calcd for $\mathrm{C}_{12} \mathrm{H}_{22} \mathrm{OS}_{2}(\%)$ : C, 58.52; H, 8.93; S, 26.03. Found: C, 58.42; H, 8.87; S, 26.10.

2,4-Dipropylthio pentanedial (5b). Yield is up to $57 \%$ after silica gel chromatography. Yellowish oil that boils undecomposed during molecular and even vacuum distillation, bp 160 ${ }^{\circ} \mathrm{C} / 1 \mathrm{~mm} \mathrm{Hg} .{ }^{1} \mathrm{H}$ NMR $\left(400 \mathrm{MHz}, \mathrm{CDCl}_{3}\right): \delta 0.99\left(\mathrm{t}, J=7.1 \mathrm{~Hz}, 6 \mathrm{H}, 2 \mathrm{CH}_{3}\right), 1.58(\mathrm{~m}, 4 \mathrm{H}$, 
$\left.\mathrm{C}_{2} \mathrm{CH}_{3}\right), 2.06$ (t, J=7.4 Hz, $\left.1 \mathrm{H}, \mathrm{H}_{2} \mathrm{C}-3\right), 2.33\left(\mathrm{~m}, 4 \mathrm{H}, 2 \mathrm{SCH}_{2}\right.$ and $\left.1 \mathrm{H}, \mathrm{H}_{2} \mathrm{C}-3\right), 3.40(\mathrm{~m}, 2 \mathrm{H}$, $\left.2 \mathrm{SC}^{*} \mathrm{H}\right), 9.33\left(\mathrm{~d},{ }^{3} J=2.3 \mathrm{~Hz} 1 \mathrm{H}, \mathrm{CHO}\right), 9.36(\mathrm{~d}, J=2.7 \mathrm{~Hz}, 1 \mathrm{H}, \mathrm{CHO}) .{ }^{13} \mathrm{C} \mathrm{NMR}\left(\mathrm{CDCl}_{3}\right): \delta$ $13.4\left(\mathrm{CH}_{3}\right), 13.6\left(\mathrm{CH}_{3}\right), 22.9,23.3,25.2,26.0,31.5\left(\mathrm{CH}_{2}\right), 50.6(\mathrm{CH}), 51.0(\mathrm{CH}), 192.5(\mathrm{CHO})$. $\mathrm{GC}-\mathrm{MS}: \mathrm{m} / \mathrm{z}(\%)=248(16)[\mathrm{M}]^{+}, 230$ (2), 205 (4) [M-Pr] $]^{+}, 187$ (3), 177 (4), 143 (24), 118 (89) $\left[\mathrm{PrSCH}_{2} \mathrm{CHO}^{+}, 101 \text { (24), } 89 \text { (26), } 76 \text { (20), } 69 \text { (19), } 43 \text { (100) [Pr] }\right]^{+}, 41$ (78), 27 (47). Anal. Calcd for $\mathrm{C}_{11} \mathrm{H}_{20} \mathrm{O}_{2} \mathrm{~S}_{2}(\%)$ : C, 53.2; H, 8.12; S, 25.81. Found: C, 52.79; H, 8.15; S, 25.81 .

2,4-Dihexylthio pentanedial (5c). Yield is up to $68 \%$ after silica gel chromatography. Yellowish oil. ${ }^{1} \mathrm{H} \mathrm{NMR}\left(\mathrm{CDCl}_{3}\right): \delta 0.89\left(\mathrm{t}, J=6.7 \mathrm{~Hz}, 6 \mathrm{H}, 2 \mathrm{CH}_{3}\right), 1.32\left(\mathrm{~m}, 12 \mathrm{H}, \mathrm{CH}_{2}\right.$ in $\left.\mathrm{C}_{6} \mathrm{H}_{13}\right), 1.52\left(\mathrm{~m}, 4 \mathrm{H}, 2 \mathrm{C}_{2} \mathrm{CH}_{2} \mathrm{~S}\right), 2.08\left(\mathrm{t}, J=7.5 \mathrm{~Hz}, 1 \mathrm{H}, \mathrm{H}_{2} \mathrm{C}-3\right), 2.37\left(\mathrm{~m}, 4 \mathrm{H}, 2 \mathrm{SCH}_{2}\right.$ and 1 $\left.\mathrm{H}, \mathrm{H}_{2} \mathrm{C}-3\right), 3.40$ (m, $\left.2 \mathrm{H}, 2 \mathrm{SCH}\right), 9.33$ (d, $\left.J=2.3 \mathrm{~Hz} 1 \mathrm{H}, \mathrm{CHO}\right), 9.35$ (d, $J=2.7 \mathrm{~Hz}, 1 \mathrm{H}$, $\mathrm{CHO}) .{ }^{13} \mathrm{C} \mathrm{NMR}\left(\mathrm{CDCl}_{3}\right): \delta 14.1\left(\mathrm{CH}_{3}\right), 22.6,28.7,29.4,29.8,29.9,31.5\left(\mathrm{CH}_{2}\right), 51.1(\mathrm{SCH})$, $192.7(\mathrm{C}=\mathrm{O})$. Anal. Calcd for $\mathrm{C}_{17} \mathrm{H}_{32} \mathrm{O}_{2} \mathrm{~S}_{2}(\%)$ : C, 61.4; H, 9.6; S, 19.3. Found: C, 61.68; H, 9.68; S, 18.98

2,4-Diheptylthio pentanedial (5d). Yield is up to $59 \%$ after silica gel chromatography. Yellowish oil. ${ }^{1} \mathrm{H} \mathrm{NMR}\left(\mathrm{CDCl}_{3}\right): \delta 0.89\left(\mathrm{t}, J=6.8 \mathrm{~Hz}, 6 \mathrm{H}, 2 \mathrm{CH}_{3}\right), 1.28\left(\mathrm{~m}, 16 \mathrm{H}, 8 \mathrm{CH}_{2}\right), 1.54$ $\left(\mathrm{m}, 4 \mathrm{H}, 2 \mathrm{CH}_{2}\right), 2.07\left(\mathrm{t}, J=7.4 \mathrm{~Hz}, 1 \mathrm{H}, \mathrm{H}_{2} \mathrm{C}-3\right), 2.36\left(\mathrm{~m}, 4 \mathrm{H}, 2 \mathrm{SCH}_{2}\right.$ and $\left.1 \mathrm{H}, \mathrm{H}_{2} \mathrm{C}-3\right), 3.40$ $(\mathrm{m}, 2 \mathrm{H}, 2 \mathrm{SC} * \mathrm{H}), 9.33$ (d, $J=2.4 \mathrm{~Hz} 1 \mathrm{H}, \mathrm{CHO}), 9.35$ (d, $J=2.7 \mathrm{~Hz}, 1 \mathrm{H}, \mathrm{CHO}) .{ }^{13} \mathrm{C}$ NMR $\left(\mathrm{CDCl}_{3}\right): \delta 14.12\left(\mathrm{CH}_{3}\right), 22.66,28.81,29.46,29.69,29.97,31.70,31.79\left(\mathrm{CH}_{2}\right), 51.10(\mathrm{SCH})$, 192.64 (C=O). Anal. Calcd for $\mathrm{C}_{19} \mathrm{H}_{36} \mathrm{O}_{2} \mathrm{~S}_{2}(\%)$ : C, 63.3; H, 10; S, 17.8. Found: C, 62.84; H, 9.87; S, 17.91 .

\section{General procedure for the synthesis of bis-2,4-dinitrophenyl hydrazones of 2,4-dialkylthio pentanedials 8a-b}

For 8a: 2,4-dibutylthio pentanedial $5 \mathbf{a}(1.8 \mathrm{mmol})$ was added to a solution of 2,4-dinitrophenyl hydrazine $(2.5 \mathrm{mmol})$ and hydrochloric acid $(2$ drops $)$ in ethanol $(123 \mathrm{~mL})$. The reaction control was carried out by TLC on silica gel plates $\left(\right.$ Silufol $\left.^{\mathrm{R}}\right)$ (acetonitrile - hexane - diethyl ether $1: 4: 1)$. On next day $0.75 \mathrm{~g}(93,4 \%)$ of bis-2,4-dinitrophenyl hydrazone of 2,4-dibutylthio pentanedial 8a were collected, $\mathrm{mp} 125{ }^{\circ} \mathrm{C}$ (recrystallized from EtOH). ${ }^{1} \mathrm{H} \mathrm{NMR}\left(\mathrm{CDCl}_{3}\right): \delta 0.87$, 0.89 (two t, $J=6.9 \mathrm{~Hz}, 6 \mathrm{H}, \mathrm{CH}_{3}$ in $\mathrm{Bu}$ ), $1.40\left(\mathrm{~m}, 4 \mathrm{H}, \mathrm{CH}_{2}\right.$ in $\left.\mathrm{Bu}\right), 1.60\left(\mathrm{~m}, 4 \mathrm{H}, \mathrm{CH}_{2}\right.$ in $\mathrm{Bu}$ ), $2.22\left(\mathrm{t}, J=6.9 \mathrm{~Hz}, 1 \mathrm{H}, \mathrm{H}_{2} \mathrm{C}-3\right), 2.52\left(\mathrm{~m}, 4 \mathrm{H}, \mathrm{CH}_{2} \mathrm{~S}\right.$ and $1 \mathrm{H}, \mathrm{H}_{2} \mathrm{C}-3$ ), 3.72 (minor) (ddd, $J=6.9$ $\mathrm{Hz}, J=6.6 \mathrm{~Hz}, J=6.9 \mathrm{~Hz}, 1 \mathrm{H}, \mathrm{CHS}$ ) and 3.82 (major) (m, $1 \mathrm{H}, \mathrm{CHS}$ ) in the 1:1.5 ratio, 7.35 (minor) and 7.47 (major) (two d, $J=6.9 \mathrm{~Hz}, 2 \mathrm{H}, \mathrm{CH}=\mathrm{N}$ ) in the 1:1.5 ratio, 7.87 (two $\mathrm{d}, J=9.7$ $\mathrm{Hz}, 2 \mathrm{H}, \mathrm{H}^{6}$ ), $8.29\left(\mathrm{~m}, 2 \mathrm{H}, \mathrm{H}^{5}\right.$ ), 9.11 and 9.13 (two d, $J=2.4 \mathrm{~Hz}, 2 \mathrm{H}, \mathrm{H}^{3}$ ), 11.05 (s, 2H, NH). ${ }^{13} \mathrm{C} \mathrm{NMR}\left(\mathrm{CDCl}_{3}\right): \delta 13.9\left(\mathrm{CH}_{3}\right), 22.2,30.4,31.8\left(\mathrm{CH}_{2}\right), 44.3(\mathrm{SCH}), 66.0\left(\mathrm{CH}_{2}\right), 116.4,123.5$, 130.2 (CH arom.), 129.5, 138.7, 145.0 (C arom.), $149.4(\mathrm{CH}=\mathrm{N}) . \mathrm{IR}(\mathrm{KBr}): 3425 \mathrm{br}(\mathrm{NH}), 1605 \mathrm{~s}$, $1580 \mathrm{~m}(\mathrm{C}=\mathrm{N}) \mathrm{cm}^{-1}$. Anal. Calcd for $\mathrm{C}_{25} \mathrm{H}_{32} \mathrm{~N}_{8} \mathrm{O}_{8} \mathrm{~S}_{2}(\%)$ : C, 47.18; H, 5.02; N, 17.59; S, 10.07. Found: C, 47.02; H, 5.14; N, 17.39; S, 9.84.

Bis-2,4-dinitrophenyl hydrazone of 2,4-dipropylthio pentanedial (8b). Yield $0.54 \mathrm{~g}, 64 \%$, mp $135{ }^{\circ} \mathrm{C}$ (recrystallized from $\mathrm{CH}_{2} \mathrm{Cl}_{2}$ ). ${ }^{1} \mathrm{H} \mathrm{NMR}\left(\mathrm{CDCl}_{3}\right): \delta 0.88$ and 0.90 (two t, $J=7.2 \mathrm{~Hz}, 6$ $\mathrm{H}, \mathrm{CH}_{3}$ in $\mathrm{Pr}$ ), $1.64\left(\mathrm{~m}, 4 \mathrm{H}, 2 \mathrm{CH}_{2}\right.$ in $\left.\mathrm{Pr}\right), 2.24\left(\mathrm{t}, J=7.2 \mathrm{~Hz}, 1 \mathrm{H}, \mathrm{H}_{2} \mathrm{C}-3\right), 2.49\left(\mathrm{~m}, 4 \mathrm{H}, 2 \mathrm{CH}_{2} \mathrm{~S}\right.$ 
and $1 \mathrm{H}, \mathrm{H}_{2} \mathrm{C}-3$ ), 3.70 (major) (m, $1 \mathrm{H}, \mathrm{SCH}$ ) and 3.80 (minor) (m, $\left.1 \mathrm{H}, \mathrm{SCH}\right), 7.39$ (minor) (d, $J$ $=7.2 \mathrm{~Hz}, 1 \mathrm{H}, \mathrm{CH}=\mathrm{N}$ ) and 7.44 (major) $(\mathrm{d}, J=6.9 \mathrm{~Hz}, 1 \mathrm{H}, \mathrm{CH}=\mathrm{N}), 7.84$ (minor), 7.86 (major) (two d, $J=9.7 \mathrm{~Hz}, 2 \mathrm{H}, \mathrm{C}^{6}$ ), 8.28 (minor) (dd, $J=9.7 \mathrm{~Hz}, J=2.4 \mathrm{~Hz}, 1 \mathrm{H}, \mathrm{C}^{5}$ ) and 8.30 (major) $\left(\mathrm{dd}, J=9.7 \mathrm{~Hz}, J=2.4 \mathrm{~Hz}, 1 \mathrm{H}, \mathrm{C}^{5}\right.$ ), 9.08 and 9.10 (two d, $J=2.4 \mathrm{~Hz}, 2 \mathrm{H}, \mathrm{C}^{3}$ ), 11.08 (s, $2 \mathrm{H}, 2$ $\mathrm{NH}) .{ }^{13} \mathrm{C} \mathrm{NMR}\left(\mathrm{CDCl}_{3}\right) \delta: 13.6\left(\mathrm{CH}_{3}\right), 22.9\left(\mathrm{CH}_{2}\right), 29.8\left(\mathrm{CH}_{2}\right), 32.6\left(\mathrm{CH}_{2}\right), 44.1(\mathrm{SCH}), 116.4$, 123.5, 130.2 (CH arom.), 129.5, 138.4, 144.9 (C arom.), 129.5, $149.4(\mathrm{CH}=\mathrm{N})$. Anal. Calcd for $\mathrm{C}_{23} \mathrm{H}_{28} \mathrm{~N}_{8} \mathrm{O}_{8} \mathrm{~S}_{2}(\%)$ : C, 45.4; H, 4.6; N, 18.4; S, 10.5. Found: C, 45.6; H, 4.9; N, 17.8 .

Alkylthioacetic aldehydes 3 were obtained by hydrolysis of acetal of alkylthioacetic aldehydes $[5,6]$.

\section{Acknowledgements}

Financial support of the Russian Foundation for Basic Research (Grant NR 08-03-00396) is gratefully acknowledged.

\section{References}

1. Hon, Y.-S.; Chang, F.-J.; Lu, L.; Lin, W.-C. Tetrahedron 1998, 54. 5233.

2. Sasaki, T.; Hayakawa, K.; Nashida, S. Tetrahedron Lett. 1980, 21, 3903.

3. Keiko, N. A.; Chuvashev, Yu. A.; Stepanova, L. G.; Bannikova, O. B.; Voronkov, M. G. Izv. Akad. Nauk SSSR, Ser. Khim. 1996, 1, 188-90; Chem. Abstr. 1996, 124, 316476.

4. Tietze, L. F.; Eicher, T. Reactionen und Synthesen im organisch-chemischen Praktikum und Forschungslaboratorium, Georg Thieme: Verlag Stuttgart, New York, 1991; S. 212. (page from russian translation).

5. Keiko, N. A.; Stepanova L. G. Izv.Akad. Nauk SSSR, Ser. Khim. 1972, 11, 2516; Chem. Abstr. 1973, 78, 71838.

6. Keiko, N. A.; Stepanova L. G. Kalikhman J. D. Voronkov M. G. Izv.Akad. Nauk USSR, Ser. Khim., 1977, 1652; Chem. Abstr. 1977, 151955.

7. Keiko, N. A.; Stepanova, L. G.; Sarapulova, G. I.; Vashchenko, A. V.; Larina, L. I.; Funtikova, E. A.; Voronkov, M. G. Russian Chemical Bulletin, Ser.Khim. International Edition, 2000, 49, 1977.

8. (a) Gunderman, K. D. Intra-Science Chem. Rept. 1972, 6, 91. (b) Gunderman, K. D.; Hnida, P. I. Angew. Chem. 1979, 91, 930.

9. Takano, S.; Inomata, K.; Samizu, K.; Tomita, S.; Yanase, M.; Suzuki, M.; Iwabuchi, Y.; Sugihara, T.; Ogasawara, K. Chem. Lett. 1989, 1283.

10. Wasilke, J.-Ch.; Obrey, S. J.; Baker, R. T.; Bazan, G. C. Chem. Rev. 2005, 105, 1001. 
11. Gorobets, E. V.; Miftakhov, M. S.; Valeev, F. A. Uspekhi Khimii 2000, 69, 1091 [Russ. Chem. Rev. 2000, 69 (Engl.Transl.)].

12. Jones, L. A.; Hancock, C. K.; Seligman, R. B. J. Org. Chem. 1961, 26, 228-232.

13. Funatcu, R.; Kurose, S.; Sato, N. Jpn. Kokai Tokkyo Koho JP 10 17,413; Chem. Abstr. 1998, 128, P 150634.

14. See for example: (a) Affaitati, P. Eur. Pat. Appl. EP 920.803, 1999; Chem. Abstr. 1999, 131, 9687. (b) Peters, J.; Spicher, G. Zentralbl. Hyg. Umweltmed. 1998, 201, 311; Chem. Abstr. 1999, 130, 322858. (c) Adabo, G. L.; Zanarotti, E.; Fonseca, R. G.; Dos Santos Crus, C. A J. Prosthet. Dent. 1999, 81, 621; Chem. Abstr. 1999, 131, 189655. (d) Goroncy-Bermes, P. Zentralbl. Hyg. Umweltmed. 1998, 201, 297. Chem. Abstr. 1999, 131, 71128.

15. See for example: (a) Goisis, G.; Yoshioka, S. A.; Braile, D. M.; Ramirez, V. D. Aparecido Artif. Organs. 1998, 22, 210; Chem. Abstr. 1998, 128, 275042. (b) Choi, H.-M.; Kim, J. H.; Shin, S. J. Appl. Polym. Sci, 1999, 73, 2691; Chem. Abstr. 1999, 131. 244542. (c) Gupta, K. C.; Jabrail, F. H. Carbohydr. Res. 2007, 342, 2244.

16. Geffard, M.; Geffard, P. PCT Int. Appl. WO 99 36, 460; Chem. Abstr. 1999, 131, P 116699.

17. (a) Comelles, J.; Moreno-Macas, M.; Vallribera, A. Arkivoc 2005 (ix), 207. (b) Perlmutter, P. Conjugate Addition Reactions in Organic Synthesis; Pergamon Press: Oxford, New York, Seoul, Tokyo, 1992, pp 63-122. (c) Kryshtal, G. V.; Zhdankina, G. M.; Astachova, I. V.; Zlotin, S. G. Izv. Akad. Nauk, Ser. Khim. 2004, 53, 647. Chem. Abstr. 2005, 142, 176422. (d) Yadav, J. S.; Geetha, V.; Subba Reddy, B. V. Synthetic Comm. 2002, 32, 3519. 